Tôhoku Math. Journ.

22(1970), 200-209.

\title{
HIGHER ORDER TANGENT BUNDLES OF PROJECTIVE SPACES AND LENS SPACES
}

\author{
HIROSHI ÔIKE
}

(Received Oct. 22, 1969)

Introduction. In [6], [7] and [8] H. Suzuki considered higher order nonimmersions of projective spaces in real affine spaces or projective spaces by means of characteristic classes, $\gamma$-operations and spin operations. In [9] C. Yoshioka obtained complete formulas of Stiefel-Whitney classes of higher order tangent bundles of complex projective spaces and Dold manifolds and he applied his results to higher order non-immersions of these spaces. In this paper, we shall study higher order tangent bundles of complex projertive spaces, quaternion projective spaces and lens spaces and compute characteristic classes of them and apply these results to higher order non-immersions of quaternion projective spaces and lens spaces.

I am grateful to Prof. H. Suzuki for his valuable suggestions.

1. Preliminaries. Let $G$ be a compact connested Lie group, $F$ be $R$ or $C$, the real or complex number field. Let $V$ be a finite dimensional $G$-vector space over $F$ and $[V]$ be a $G$-isomorphism class of $V$, then the dimension of $V$ is said to be the degree of $[V]$.

We denote $k$-fold symmetric product over $F$ of $V$ by $O^{k} V$, then $G$ acts on $O^{k} V$ as follows :

$$
g\left(v_{1} \bigcirc v_{2} \bigcirc \cdots \bigcirc v_{k}\right)=g v_{1} \bigcirc g v_{2} \bigcirc \cdots \bigcirc g v_{k} \text { for } g \in G,
$$

where $v_{1} \bigcirc v_{2} \bigcirc \cdots \bigcirc v_{k}$ is the image of $v_{1} \otimes v_{2} \otimes \cdots \otimes v_{k}$ by the symmetrization operator from $k$-fold tensor product $\otimes^{k} V$ to $O^{k} V$. Thus $O^{k} V$ is a $G$-vector space. We have the following lemma:

LEMMA 1.1. Let $V$ be one dimensional G-vector space, then $O^{k} V$ is isomorphic to $\otimes^{k} V$.

Let $M_{F}(G)$ be a semiring which consists of all $G$-isomorphism classes of finite dimensional $G$-vector spaces over $F$. The sum and product in $M_{r}(G)$ are 
induced by direct sum and tensor product of finite dimensional $G$-vector spaces over $F$. We define $O^{k}[V]$ for $[V] \in M_{F}(G)$ as follows

$$
O^{k}[V]=\left[O^{k} V\right],
$$

then $O^{k}$ induces an operation of $M_{F}(G)$ having following properties :

i) $O^{\circ}(x)=1, O^{1}(x)=x$ for $x \in M_{F}^{\prime}(G)$,

ii) $O^{k}(x+y)=\sum_{i+j=k} O^{i}(x) O^{j}(y)$ for $x, y \in M_{F}(G)$,

iii) $O^{k}(x)=x^{k}$ for $x \in M_{F}(G)$; of degree 1 .

Let $R_{F}(G)$ be the ring completion of $M_{F}(G)$ and $\theta: M_{F}(G) \longrightarrow R_{F}(G)$ be the natural inclusion map.

Then the above $O^{k}$ can be extended to $R_{F}(G)$ and the properties i), ii) are preserved in $R_{F}(G)$ too, but the properties iii) is satisfied in $\operatorname{Im} \theta$ only.

Now, let $r, c, \psi_{c}^{-1}$ be the following operations

$$
\begin{aligned}
& r: R_{C}(G) \longrightarrow R_{R}^{\prime}(G) \text { realification, } \\
& c: R_{R}(G) \longrightarrow R_{C}(G) \text { complexification, } \\
& \psi_{c}^{-1}: R_{C}\left(G \longrightarrow R_{C}(G)\right. \text { complex conjugation. }
\end{aligned}
$$

Then we have the following lemma (see [1]).

LEMMA 1.2. i) $r$ is a group homomorphism and $c$ and $\psi_{c}^{-1}$ are ring homomorphisms:

ii) $r c=2, c r=1+\psi_{c}^{-1}$.

iii) $c$ is injective.

iv) $c O^{k}=()^{k} c$.

Next, let $t$ be an indeterminate and let $1+R_{F}(G)[[t]]^{+}$be the multiplicative group which consists of all units in the ring $R_{F}^{\prime}(G)[[t]]$.

We define a map

$$
O_{t}: R_{F}(G) \longrightarrow 1+R_{F}(G)[[t]]^{+}
$$

by

$$
O_{t}(x)=\sum_{k=0}^{\infty} O^{k}(x) t^{k} \quad \text { for } \quad x \in R_{r}(G)
$$


Then, by Lemma 1.2 , we have

$$
O_{t}(c x)=\sum_{k=0}^{\infty}\left(c O^{k}(x)\right) t^{k} \quad \text { for } \quad x \in R_{R}(G)
$$

The following theorem is described in [2].

THEOREM 1.3. $R_{C}(U(1))$ equals $Z\left[Z, Z^{-1}\right]$, where $z$ is a $U(1)$-isomorphism class of degree 1 such that $U(1)$ acts on one dimensional vector space $C$ (the field of complex numbers) as follows: $\left(e^{i \theta}, w\right) \longmapsto e^{i} \cdot w$ for $e^{i \theta} \in U(1)$, $w \in C$. And $z^{-1}=\psi_{C}^{-1} z$.

Let $\eta=r z-2 \in R_{R}(U(1))$, then we have the following lemma :

LEMMA 1.4 . i) $\psi_{R}^{k}(\eta)=r z^{k}-2, \psi_{R}^{0}(\eta)=0, \psi_{R}^{-k}(\eta)=\psi_{R}^{k}(\eta)$,

ii) $\eta^{j}=\sum_{i=1}^{j}(-1)^{j-i}\left(\begin{array}{c}2 . j \\ j-i\end{array}\right) \psi_{R}^{i}(\eta), \quad$ iii) $\quad \psi_{R}^{k}(\eta)=\sum_{j=1}^{k} A_{j}^{k} \eta^{j}$,

iv) $\psi_{R}^{i}(\eta) \psi_{R}^{j}(\eta)=\psi_{R}^{i+j}(\eta)+\psi_{R}^{j-i}(\eta)-2\left(\psi_{R}^{i}(\eta)+\psi_{R}^{j}(\eta)\right)$,

where $\psi_{R}^{k}$ is the real Adams operation (see [1]) and

$$
A_{j}^{k}=\frac{2}{(2, j) !} \prod_{i=1}^{j-1}\left(k^{2}-i^{2}\right)=\frac{k}{j}\left(\begin{array}{c}
k+j-1 \\
2 j-1
\end{array}\right)
$$

Since proofs require only tedious calculations, we omit them.

2. Calculations and applications. In the first place, we prove the following key lemma :

LEMMA 2. 1.

$$
O^{k}((n+1) r z)=\sum_{j \neq 0}^{\left[\frac{k-1}{3}\right]}\left(\begin{array}{c}
n+j \\
j
\end{array}\right)\left(\begin{array}{c}
n+k-j \\
k-j
\end{array}\right) \psi_{R}^{k-2 j}(\eta)+\left(\begin{array}{c}
2 n+k+1 \\
k
\end{array}\right) .
$$

PROOF. Since $z$ and $z^{-1}$ are of degree 1

$$
\begin{aligned}
O_{t}((n+1) c r z) & =\left(O_{t}\left(z+z^{-1}\right)\right)^{n+1}=(1-z t)^{-(n+1)}\left(1-z^{-1} t\right)^{-(n+1)} \\
& =1+\sum_{k=1}^{\infty} \sum_{i+j=k}\left(\begin{array}{c}
n+i \\
i
\end{array}\right)\left(\begin{array}{c}
n+j \\
j
\end{array}\right) z^{j-i} t^{k}
\end{aligned}
$$


Hence

$$
c O^{k}((n+1) r z)=\sum_{i+j=k}\left(\begin{array}{c}
n+i \\
i
\end{array}\right)\left(\begin{array}{c}
n+j \\
j
\end{array}\right) z^{j-i}
$$

By Lemma 1.2 and 1.4, the proof is completed.

Let $h_{c}$ be the canonical complex line bundle over an $n$-dimensional complex projective space $C P^{n}$.

By Suzuki's theorem (1.1) of [7], the $k$-th order tangent bundle of an $n$-dimensional complex projective space $C P^{n}$ is given by

$$
\tau_{k}\left(C P^{n}\right)=O^{k}\left((n+1) r h_{C}\right)-O^{k-1}\left((n+1) r h_{c}\right)-1 \text {. }
$$

Hence by Lemma 2.1 we have

THEOREM 2.2. Let $y=r h_{c}-2$, then

$$
\begin{aligned}
\tau_{k}\left(C P^{n}\right)+1= & \sum_{j=0}^{\left[\frac{k-1}{2}\right]}\left(\begin{array}{c}
n+j \\
j
\end{array}\right)\left\{\left(\begin{array}{c}
n+k-j \\
k-j
\end{array}\right) \psi_{R}^{k-2 j}(y)\right. \\
& \left.-\left(\begin{array}{c}
n+k-j-1 \\
k-j-1
\end{array}\right) \psi_{R}^{k-2 j-1}(y)\right\}+\left(\begin{array}{c}
2 n+k \\
k
\end{array}\right) .
\end{aligned}
$$

For example, by Lemma 1.4 we have

$$
\begin{aligned}
\tau_{\mathrm{s}}\left(C P^{n}\right)+1= & \left(\begin{array}{c}
n+2 \\
2
\end{array}\right) y^{2}+\left(\begin{array}{c}
2 n+3 \\
2
\end{array}\right) y+\left(\begin{array}{c}
2 n+2 \\
2
\end{array}\right) \\
\tau_{3}\left(C P^{n}\right)+1= & \left(\begin{array}{c}
n+3 \\
3
\end{array}\right) y^{3}+(2 n+5)\left(\begin{array}{c}
n+2 \\
2
\end{array}\right) y^{2}+6\left(\begin{array}{c}
2 n+4 \\
3
\end{array}\right) y+\left(\begin{array}{c}
2 n+3 \\
3
\end{array}\right) \\
\tau_{4}\left(C P^{n}\right)+1= & \left(\begin{array}{c}
n+4 \\
4
\end{array}\right) y^{4}+(2 n+7)\left(\begin{array}{c}
n+3 \\
3
\end{array}\right) y^{3}+3(2 n+5)\left(\begin{array}{c}
n+3 \\
3
\end{array}\right) y^{2} \\
& +2\left(\begin{array}{c}
2 n+5 \\
4
\end{array}\right) y+\left(\begin{array}{c}
2 n+4 \\
4
\end{array}\right)
\end{aligned}
$$

Next we calculate $k$-th order Pontrjagin class $P\left(\tau_{k}\left(C P^{n}\right)\right)$ of $C P^{n}$.

Let $x$ be a generator of $H^{2}\left(C P^{n} ; Z\right)$. Then we can see easily that the Pontrjagin class of $r h_{C}^{j}$ is given by 


$$
P\left(\psi_{R}^{j}(y)\right)=P\left(r h_{c}^{j}\right)=1+j^{2} x^{2}
$$

Since $H^{*}\left(C P^{n} ; Z\right)$ has no 2 -torsion, we have the following corollary :

\section{COROLLARY 2.3.}

$$
P\left(\tau_{k}\left(C P^{n}\right)\right)=\sum_{j=0}^{\left[\frac{k-1}{2}\right]}\left\{\frac{\left(1+(k-2 j)^{2} x^{2}\right)^{\left(\begin{array}{c}
n+k-j \\
k-j
\end{array}\right)}}{\left(1+(k-2 j-1)^{2} x^{2}\right)^{\left(\begin{array}{c}
n+k-j-1 \\
k-j-1
\end{array}\right)}}\right\}^{\left(\begin{array}{c}
n+j \\
j
\end{array}\right)} .
$$

Now we calculate higher order tangent bundles and higher order charactris: tic classes of the quaternion projective space $H P^{n}$.

Let $\pi: C P^{2 n+1} \longrightarrow H P^{n}$ be the canonical $S^{2}$-bundle and let $h_{H}$ be the canonical complex plane bundle over the quaternion projective space $H P^{n}$. We have following commutative diagram

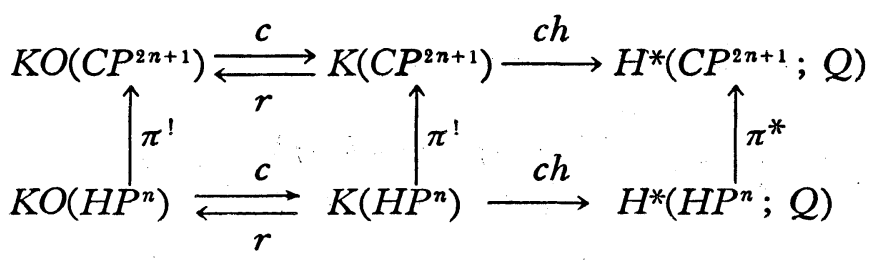

where all vertical arrows are ring monomorphisms (see [4]).

The following lemma is easily seen by means of results described in [4].

LEMMA 2.4. i) Complex conjugation $\psi_{c}^{-1}: K\left(H P^{n}\right) \rightarrow K\left(H P^{n}\right)$ is identity. ii) First order tangent bundle $\tau\left(H P^{n}\right)=(n+1) r h_{H}-\frac{\left(r h_{H}\right)^{2}}{4}$. iii) $\pi^{!} r h_{H}=2 r h_{C}$. iv) $\pi^{!} h_{H}=h_{C}+h_{C}^{-1}$. v) $c r h_{H}=2 h_{H}$. vi) $\pi^{*} q=x^{2}$, where $q$ and $x$ are the generators of $H^{4}\left(H P^{n} ; Q\right)\left(\right.$ or $\left.H^{4}\left(H P^{n} ; Z\right)\right)$ and $H^{2}\left(C P^{2 n+1} ; Q\right)\left(\right.$ or $H^{2}\left(C P^{2 n+1}\right.$; $Z)$ ) respectively. vii) Restriction of $c: K O\left(C P^{2 n+1}\right) \rightarrow K\left(C P^{2 n+1}\right)$ to free part of $K O\left(C P^{2 n+1}\right)$ and $c: K O\left(H P^{n}\right) \rightarrow K\left(H P^{n}\right)$ are injective.

By this lemma we have following fact

$$
\pi^{!} c\left(\tau\left(H P^{n}\right)+1\right)=2(n+1)\left(h_{C}+h_{c}^{-1}\right)-h_{C}^{2}-h_{c}^{-2}-1 .
$$

Thus

$$
O_{t}\left(\pi^{1} c\left(\tau\left(H P^{n}\right)+1\right)\right)=O_{t}\left(2(n+1)\left(h_{c}+h_{c}^{-1}\right)\right)\left(1-h_{c}^{2} t\right)\left(1-h_{c}^{-2} t\right)(1-t)
$$


Hence

$$
\pi^{!} \tau_{k}\left(H P^{n}\right)+1=O^{k}-O^{k-3}-\left(r h_{C}^{2}+1\right)\left(O^{k-1}-O^{k-2}\right),
$$

where $O^{s}=O^{s}\left(2(n+1) r h_{c}\right)$.

By Lemma 2.1 and iv) of Lemma 1.4, we have

THEOREM 2.5 .

$$
\begin{aligned}
\pi^{1} \tau_{k}\left(H P^{n}\right)+1 & =\sum_{j=0}^{\left[\frac{k-1}{2}\right]}\left(\begin{array}{c}
2 n+1+j \\
j
\end{array}\right)\left(\begin{array}{c}
2 n+1+k-j \\
k-j
\end{array}\right) \psi_{R}^{k-2 j}(y) \\
& -\sum_{j=0}^{\left[\frac{k}{2}\right]-1}\left(\begin{array}{c}
2 n+1+j \\
j
\end{array}\right)\left(\begin{array}{c}
2 n+1+k-1-j \\
k-1-j
\end{array}\right)\left(\psi_{R}^{k+1-2 j}(y)+\psi_{R}^{k-1-2 j}(y)+\psi_{R}^{k-3-2 j}(y)\right) \\
& +\sum_{j=0}^{\left[\frac{k-1}{2}\right]-1}\left(\begin{array}{c}
2 n+1+j \\
j
\end{array}\right)\left(\begin{array}{c}
2 n+1+k-2-j \\
k-2-j
\end{array}\right)\left(\psi_{R}^{k-2 j}(y)+\psi_{R}^{k-2-2 j}(y)+\psi_{R}^{k-4-2 j}(y)\right) \\
& -\left[\begin{array}{l}
{\left[\frac{k}{2}\right]-2} \\
j=0
\end{array}\right)\left(\begin{array}{c}
2 n+1+j \\
j \\
k-3-j
\end{array}\right) \psi_{R}^{k-3-2 j}(y) \\
+ & (-1)^{k}\left(\begin{array}{c}
2 n+1+\left[\frac{k-1}{2}\right] \\
{\left[\frac{k-1}{2}\right]}
\end{array}\right)^{2} \psi_{R}^{2}(y)+\left(\begin{array}{c}
4 n+k \\
k
\end{array}\right)
\end{aligned}
$$

where $y=r h_{c}-2$.

Next we calculate $k$-th order Stiefel-Whitney class $W\left(\tau_{k}\left(H P^{n}\right)\right)$.

$$
W\left(\psi_{R}^{j}(y)\right)=W\left(r h_{C}^{j}\right)=C\left(h_{C}^{j}\right) \bmod 2=1+j \bar{x},
$$

where $\vec{x}=x \bmod 2$, and $C\left(h_{c}^{j}\right)$ is Chern class of $h_{c}^{j}$.

Hence,

$$
W\left(\psi_{R}^{j}(y)\right)=\left\{\begin{array}{l}
1 \text { for even } j \\
1+\vec{x} \text { for odd } j
\end{array}\right.
$$

In general, for an odd positive integer $l$ 


$$
\sum_{j=0}^{\frac{l-1}{2}}\left(\begin{array}{c}
2 n+1+j \\
j
\end{array}\right)\left(\begin{array}{c}
2 n+1+l-j \\
l-j
\end{array}\right)=\frac{1}{2}\left(\begin{array}{c}
4 n+l+3 \\
l
\end{array}\right)
$$

and

$$
\left(\begin{array}{c}
4 n+l+3 \\
l
\end{array}\right) \equiv 0(\bmod 4)
$$

Thus, by Theorem 2.5,

$$
\bar{\pi}^{*} W\left(\tau_{k}\left(H P^{n}\right)\right)= \begin{cases}(1+\bar{r})^{2 N_{0}(n, k)} & \text { for odd } k \\ (1+\bar{x})^{-2 N_{e}(n, k)} & \text { for even } k,\end{cases}
$$

where $\bar{\pi}^{*}=\pi^{*} \bmod 2$ is injective and

$$
\begin{aligned}
& N_{0}(n, k)=\frac{1}{4}\left(\begin{array}{c}
4 n+k+3 \\
k
\end{array}\right)+\frac{3}{4}\left(\begin{array}{c}
4 n+k+1 \\
k-2
\end{array}\right) \text { for odd } k \\
& N_{e}(n, k)=\frac{3}{4}\left(\begin{array}{c}
4 n+k+2 \\
k-1
\end{array}\right)+\frac{1}{4}\left(\begin{array}{c}
4 n+k \\
k-3
\end{array}\right) \text { for even } k
\end{aligned}
$$

Now $(1+\bar{x})^{2}=1+\bar{x}^{2}=1+\bar{\pi}^{*} \bar{q}=\bar{\pi}^{*}(1+\bar{q})$, where $\bar{q}=q \bmod 2$. Therefore THEOREM 2.6.

$$
W\left(\tau_{k}\left(H P^{n}\right)\right)= \begin{cases}(1+\bar{q})^{N_{0}(n, k ;} & (k: \text { odd }) \\ (1+\bar{q})^{-N_{e}(n, k)} & (k: \text { even }) .\end{cases}
$$

Let $\delta_{0}(n, k), \sigma_{0}(n, k), \delta_{e}(n, k), \sigma_{e}(n, k)$ be following integers

$$
\begin{aligned}
& \delta_{0}(n, k)=\max \left\{1 \leqq i \leqq n ;\left(\begin{array}{c}
N_{0}(n, k) \\
i
\end{array}\right) \neq 0(\bmod 2)\right\}, \\
& \sigma_{0}(n, k)=\max \left\{1 \leqq i \leqq n ;\left(\begin{array}{c}
N_{0}(n, k)+i-1 \\
i
\end{array}\right) \neq 0(\bmod 2)\right\} \\
& \delta_{e}(n, k)=\max \left\{1 \leqq i \leqq n ;\left(\begin{array}{c}
N_{e}(n, k) \\
i
\end{array}\right) \neq 0(\bmod 2)\right\} \\
& \sigma_{e}(n, k)=\max \left\{1 \leqq i \leqq n ;\left(\begin{array}{c}
N_{e}(n, k)+i-1 \\
i
\end{array}\right) \neq 0(\bmod 2)\right\}
\end{aligned}
$$


By this theorem and Theorem (1.1) of [6], we have the following corollary.

COROLLARY 2.7. When $k$ is odd, if $m$ is an integer such that $-4 \delta_{0}(n, k)<m<4 \sigma_{0}(n, k)$ and when $k$ is even, if $m$ is an integer such that $-4 \sigma_{\rho}(n, k)<m<4 \delta_{\rho}(n, k)$, then

$$
H P^{n} q_{k} R^{\left(\begin{array}{c}
4 n+k \\
k
\end{array}\right)-1+m} .
$$

Since Pontrjagin class of $\tau_{k}\left(H P^{n}\right)$ can be easily calculated in similar manner of Corollary 2. 3 and Theorem 2.6, we omit its calculation.

Now we consider higher order tangent bundles, characteristic classes and non-immersions of lens space.

Let $p$ be an integer larger than one and let $L^{\prime \prime}(p)=L(p ; 1, \cdots, 1)$ be $2 n+1$-dimensional lens space of mod $p$. The following fact is well known (see[3])

$$
\tau\left(L^{n}(p)\right)+1=(n+1) \pi \cdot r h_{C},
$$

where $\pi: L^{n}(p) \longrightarrow C P^{n}$ is canonical $S^{1}$-bundle.

By Lemma 2.1 we have

THEOREM 2.8 .

$$
\tau_{k}\left(L^{n}(p)\right)+1=\sum_{j=0}^{\left[\begin{array}{c}
k-1 \\
2
\end{array}\right]}\left(\begin{array}{c}
n+j \\
j
\end{array}\right)\left(\begin{array}{c}
n+k-j \\
k-j
\end{array}\right) \psi_{k}^{k-2 j}(\sigma)+\left(\begin{array}{c}
2 n+1+k \\
k
\end{array}\right),
$$

where $\sigma=\pi^{!}(y)$.

We have the following corollary on $k$-th order characteristic classes of $L^{n}(p)$. H. Suzuki informed me of this result.

Corollary 2.9. If $p$ is odd, the Pontrjagin class of $\tau_{k}\left(L^{n}(p)\right)$ is given by

$$
P\left(\tau_{k}\left(L^{n}(p)\right)\right)=\prod_{j=0}^{\left[\begin{array}{c}
\frac{k-1}{2} \\
j
\end{array}\right.}\left(1+(k-2 j)^{2} u^{2}\right)^{\left(\begin{array}{c}
n+j \\
j
\end{array}\right)\left(\begin{array}{c}
n+k-j \\
k-j
\end{array}\right)},
$$

and if $p$ is even, the Stiefel-Whitney class of $\tau_{k}\left(L^{n}(p)\right)$ is given by

$$
W\left(\tau_{k}\left(L^{n}(p)\right)\right)=\left\{\begin{array}{cl}
\left.(1+\bar{u})^{\frac{1}{2}(2 n+1+k}\right) & (k: \text { odd }) \\
1 & (k: \text { even }),
\end{array}\right.
$$


where $u$ is generator of $H^{2}\left(L^{n}(p) ; Z\right)$ and $\bar{u}=u \bmod 2$.

PROOF. $P\left(\psi_{R}^{j}\left(\sigma^{*}\right)\right)=\pi^{*}\left(1+j^{2} x^{2}\right)=1+j^{2}\left(\pi^{*} x\right)^{2}=1+j^{2} u^{2}$. Since $p$ is odd, $H^{*}\left(L^{n}(p) ; Z\right)$ has ro 2 -torsion element (see 5.2. Theorem of [5]).

Thus, the proof for the Pontrjagin class is completed.

$$
W\left(\psi_{R}^{j}(\sigma)\right)=1+j \bar{\pi}^{*} \bar{x}=1+j \bar{u}=\left\{\begin{array}{c}
1+\bar{u}(j: \text { odd }) \\
1 \text { (j: even) } .
\end{array}\right.
$$

This completes the proof for the Stiefel-Whitney class.

Next by Theorem 2.8 and Corollary 2.9 , we consider $k$-th order nonimmersions of $L^{n}(3)$ in real affine spaces.

$\pi^{!} h_{C}^{3}=1$ in $K\left(L^{n}(3)\right)$ and by Lemma 1.2 and Theorem $1.3, r z^{-1}=r z$. Hence $\psi_{k}^{2}\left(\sigma^{\circ}\right)=\psi_{R}^{1}\left(\sigma^{\circ}\right)=\sigma$.

Let $K_{n, k}^{0}, K_{n, k}^{1}$, and $K_{n, k}^{2}$ be the following integers

$$
\begin{aligned}
& K_{n, k}^{0}=\sum_{j=0}^{\left[\frac{k-1}{6}\right]}\left(\begin{array}{c}
n+3 j \\
3 j
\end{array}\right)\left(\begin{array}{c}
n+k-3 j \\
k-3 j
\end{array}\right), \\
& K_{n, k}^{1}=\sum_{j=0}^{\left[\frac{k-3}{6}\right]}\left(\begin{array}{c}
n+3 j+1 \\
3 j+1
\end{array}\right)\left(\begin{array}{c}
n+k-3 j-1 \\
k-3 j-1
\end{array}\right), \\
& K_{n, k}^{2}=\sum_{j=0}^{\left[\frac{k-5}{6}\right]}\left(\begin{array}{c}
n+3 j+2 \\
3 j+2
\end{array}\right)\left(\begin{array}{c}
n+k-3 j-2 \\
k-3 j-2
\end{array}\right) .
\end{aligned}
$$

Then by Thsorem 2.7 we have

$$
\tau_{n}^{n}\left(L^{n}(3)\right)=\left(K_{n, k}^{\alpha}+K_{n, k}^{\beta}\right) \sigma \text { for } \alpha+\beta \equiv k(\bmod 3) \& \alpha \neq \beta,
$$

where $\tau_{k}^{n}$ is stable class of $\tau_{i c}\left(L^{n}(3)\right)$. $L^{n}(3)$.

We employ $k$-th order Pontrjagin classes on $k$-th oxder non-immersions of

H. Suzuki informed me that this manner is more convenient on this problem than $\gamma$-operations.

$$
P\left(\tau_{k}\left(L^{n}(3)\right)\right)=\left(1+u^{2}\right)^{K_{n k}^{\alpha}+K_{n, k}^{\beta}} \text { for } \alpha+\beta \equiv k(\operatorname{mot} 3) \& \alpha \neq \beta .
$$

Let $d_{P}(n, k), s_{P}(n, k)$ be the following integers 


$$
\begin{aligned}
& d_{P}(n, k)=\max \left\{m ; 1 \leqq m \leqq \frac{n}{2},\left(\begin{array}{c}
K_{n, k}^{\alpha}+K_{n, k}^{\beta} \\
m
\end{array}\right) \neq 0(\bmod 3)\right\}, \\
& s_{P}(n, k)=\max \left\{m ; 1 \leqq m \leqq \frac{n}{2},\left(\begin{array}{c}
K_{n, k}^{\alpha}+K_{n, k}^{\beta}+m-1 \\
m
\end{array}\right) \neq 0(\bmod 3)\right\} .
\end{aligned}
$$

By similar argument on Pontrjagin classes to Theorem (1.1) of [6], we have the following theorem.

THEOREM 2.10. For $-2 d_{P}(n, k)<m<2 s_{P}(n, k)$

$$
\left.L^{n}(3) \neq{ }_{k} R^{(2 n+1+k}\right)-1+m .
$$

\section{REFERENCES}

[1] J. F. ADAMS, Vector fields on spheres, Ann. of Math., 75(1962), 603-632.

[2] D. Husemoller, Fiber Bundle, McGraw-Hill Book Company, New York, 1966.

[3] T. KAMBE, The structure of $K_{\Lambda}$-ring of lens space and their applications, J. Math. Soc. Japan, 18(1966), 135-146.

[ 4 ] B. J. SANDIRSON, Immersions and embeddings of projective scaces, Proc. London Math. Soc., (3)14(1964), 137-153.

[5] N. E. Ster.nrod, Cohomology Operations, Princeton University Press, 1962.

[6] H. SUZUKI, Bounds for dimensions of odd order non-singular immersions of $R P^{n}$, Trans. Amer. Math. Soc., 121(1966), 269-275.

[7] H. SUZUKI, Characteristic classes of some higher order tangent bundles of complex projective spaces, J. Math. Soc. Japan, 18(1966), 386-393.

[8] H.SUZUKI, Higher order non-singular immersions in projective spaces, Quart. J. Math. Oxford (2), 20(1969), 33-44.

[9] C. YoshiokA, On the higher order non-singular immersions, Sci. Rep. Niigata Univ. Ser. A, 5(1967), 23-30. 